\title{
Distribuição de Género em Cargos de Liderança na Área Médica em Portugal: O Exemplo das Candidaturas aos Órgãos da Ordem dos Médicos 2017-2019
}

\author{
Gender Distribution in Medical Leadership Roles in \\ Portugal: The Example of Candidacy to Bodies of the \\ Portuguese Medical Association 2017-2019
}

\author{
Miguel CABRAL ${ }^{1,2}$, Margarida PAIXÃO ${ }^{1}$, Andreia LEITE $\triangle^{1,3}$ \\ Acta Med Port 2021 May;34(5):342-346 - https://doi.org/10.20344/amp.12955
}

\section{RESUMO}

Introdução: A igualdade de género constitui um dos objetivos de desenvolvimento sustentável. Uma manifestação de desigualdade de género é a baixa participação de mulheres em cargos de liderança. Em Portugal, são escassos os estudos sobre a desigualdade de género na liderança na área médica. Assim, o presente trabalho pretendeu analisar a distribuição de género em candidaturas aos órgãos regionais da Ordem dos Médicos.

Material e Métodos: Foram extraídos da Revista da Ordem dos Médicos (número 175) os dados dos candidatos aos órgãos regionais da Ordem dos Médicos (mandato de 2017 - 2019). Obtiveram-se as percentagens de mulheres candidatas, globalmente, por lista, regiões e cargos. Calcularam-se razões observado-versus-esperado por secção regional e intervalos de confiança a 95\% assumindo uma distribuição de Poisson. Foi realizada análise de sensibilidade, excluindo os candidatos a suplentes.

Resultados: Trinta e sete por cento dos candidatos eram médicas (variação por região: 29\% - 51\%). A nível nacional a razão observado-versus-esperado foi de 0,74 (intervalo confiança a $95 \%$ : 0,58; 0,92), principalmente influenciada pela razão da região Sul de 0,58 (intervalo confiança a 95\%: 0,41;0,80). Existiu uma predominância de mulheres nas candidaturas para suplentes e secretário $(56 \%$ e $54 \%$ respetivamente)

Discussão: A diferença entre géneros é particularmente acentuada na região Sul, na frequência e tipo de cargos a que se candidatam. As razões apontadas na literatura relacionam-se com a maternidade, o papel social da mulher e perceções sobre o desempenho dos cargos de lideraça. Este estudo é limitado à análise de um tipo de liderança e um momento eleitoral, sendo necessárias análises mais abrangentes.

Conclusão: Houve menor participação do que o expectável por parte das médicas no processo eleitoral da Ordem dos Médicos. Quando participam, as mulheres tendem a fazê-lo em cargos de menor relevância ou com menos potencial para eleição (secretário ou suplente). É necessário aprofundar o estudo e introduzir medidas de combate à desigualdade de género em cargos de liderança.

Palavras-chave: Identidade de Género; Liderança; Portugal; Sexismo; Sociedades Médicas

\section{ABSTRACT}

Introduction: Gender equality is one of the sustainable development goals. Low participation of women in leadership roles is an example of gender inequality. In Portugal, there are few studies regarding gender inequality in medical leadership roles. Therefore, we aimed to analyse gender distribution of candidates to regional bodies of the Portuguese Medical Association.

Material and Methods: We extracted data from the candidates to the regional bodies of the Portuguese Medical Association (2017 - 2019 mandate) from the Association's magazine (issue number 175). We calculated the percentage of women candidates, overall and stratified by list, region and roles. We obtained observed-vs-expected ratios overall and by region, and respective $95 \%$ confidence intervals, assuming a Poisson distribution. Finally, we conducted a sensitivity analysis, excluding substitute candidates.

Results: Women accounted for $37 \%$ of the candidates (regional variation: $29 \%-51 \%$ ). The national observed-vs-expected ratio was 0.74 (95\% confidence interval: $0.58 ; 0.92)$, mainly driven by the ratio from the South Region: 0.58 (95\% confidence interval: $0.41 ; 0.80)$. Women ran mainly for alternate candidates and secretary positions ( $56 \%$ and $54 \%$ respectively).

Discussion: Gender differences were identified, particularly in the South, regarding the frequency and type of candidacy. Previous works have identified maternity, the social role of women and perceptions regarding the leadership roles as possible reasons to explain such differences. Our analysis is limited to specific leadership roles and an election moment; further studies should be pursued.

Conclusion: We identified a lower than expected participation of women in the elections for the Portuguese Medical Association. When they run, women are found mainly in less relevant positions or with less potential to be elected (secretary or alternate candidate). A deeper understanding and measures to fight gender inequality in leadership roles are required.

Keywords: Gender Identity; Leadership; Portugal; Sexism; Societies, Medical

\section{INTRODUÇÃO}

A igualdade de género é um dos valores fundadores da Declaração Universal dos Direitos Humanos e da União

Europeia, sendo, desde os anos 90, um dos pontos altos da agenda europeia. ${ }^{1,2} \mathrm{~A}$ importância de atingir a igualdade

1. Unidade de Saúde Pública António Luz. Agrupamento de Centros de Saúde da Amadora. Damaia. Portugal.

2. World Health Organization Collaborating Centre for Health Policy, Governance and Leadership in Europe. Università Cattolica del Sacro Cuore. Rome. Italy.

3. Centro de Investigação em Saúde Pública. Escola Nacional de Saúde Pública. Universidade NOVA de Lisboa. Lisboa. Portugal.

$\triangle$ Autor correspondente: Andreia Leite. andreia.leite@ensp.unl.pt

Recebido: 14 de outubro de 2019 - Aceite: 20 de janeiro de 2020 - First published: 09 de dezembro de 2020 - Online issue published: 03 de maio de 2021 Copyright $\odot$ Ordem dos Médicos 2021 
de género é também contemplada nos objetivos de desenvolvimento sustentável das Nações Unidas, mais concretamente no objetivo número cinco. ${ }^{3}$

Uma das manifestações da desigualdade de género é a fraca participação das mulheres em cargos de liderança. Esta questão tem sido discutida com particular foco em cargos de liderança na área política e no setor económico. ${ }^{1}$ Em Portugal, estudos realizados em empresas indicam que a representação das mulheres nos órgãos de gestão se encontra abaixo dos países nórdicos. ${ }^{4}$ Um estudo das empresas que integram o PSI-20 demonstrou que em 2016, das 19 empresas analisadas, seis não tinham mulheres no conselho de administração, e a participação feminina máxima era de 33,3\%. ${ }^{5}$ Uma análise mais recente indica a baixa feminização em cargos de nomeação política em todas as áreas governativas. ${ }^{6}$

Estudos realizados nos Estados Unidos da América revelam que apenas $18 \%$ dos diretores executivos dos hospitais, $15 \%$ dos responsáveis de departamento das faculdades médicas e $16 \%$ dos diretores de faculdades médicas são mulheres. Estes valores verificam-se apesar de o número de graduados do sexo feminino ter vindo a aumentar, e metade ou mais dos estudantes que terminam a faculdade serem atualmente mulheres. ${ }^{7}$ Em Portugal, a proporção de mulheres na profissão médica tem aumentado nas últimas décadas, com $40,2 \%$ de médicos do sexo feminino em 1991, 50,2\% em 2010 e 55,3\% em 2018. ${ }^{8}$ Como tal, seria expectável começar a ver um maior número de cargos de liderança a serem ocupados por médicas. No entanto, tal como em outros países, esta tendência de maior percentagem de mulheres na medicina não se tem traduzido em maior percentagem de mulheres em cargos de liderança. ${ }^{7,9,10}$ Também parece ser este o caso na escassa literatura que aborda esta área específica no nosso país. No final do século passado, apenas $20 \%$ dos docentes era do género feminino na Faculdade de Medicina da Universidade do Porto. Num dos grandes hospitais de Lisboa, 52\% dos médicos eram mulheres, mas dos diretores de serviços apenas $17 \%$ eram mulheres. ${ }^{11}$ Contudo, não são conhecidos estudos de abrangência nacional relativos à desigualdade de género em cargos de liderança na área médica (incluindo hospitais e faculdades médicas). A Ordem dos Médicos (OM) congrega atualmente todos os médicos a trabalhar em Portugal, ${ }^{12}$ e, ainda que seja uma entidade nacional, está dividida estruturalmente em três secções regionais: Norte, Centro e Sul, encontrando-se as Regiões Autónomas incluidas nesta última. ${ }^{12}$ Enquanto instituição democrática, a OM é liderada por estruturas constituídas por indivíduos eleitos após um processo eleitoral aberto a todos os médicos. Contudo, a função de liderança de maior destaque, a figura de bastonário, nunca foi ocupada por uma médica. ${ }^{12}$ Dada a inexistência de estudos de âmbito nacional relativos à distribuição de género em cargos de liderança na área médica, o objetivo deste trabalho é descrever a distribuição por género das candidaturas às diferentes listas para os órgãos regionais das três secções da OM (Norte, Centro e Sul) para o triénio 2017-2019. Preten- de-se, igualmente, identificar diferenças entre géneros nas candidaturas relativamente a posições dentro das candidaturas.

\section{MATERIAL E MÉTODOS}

Procedeu-se à extração dos dados para análise da Revista da Ordem dos Médicos $n^{\circ}$. 175, de dezembro de $2016 .{ }^{13}$ Foram recolhidos o nome de todos os candidatos, órgão e cargo a que se candidatavam, assim como a lista e secção regional pelo qual o faziam. O género dos candidatos foi identificado com base no nome. Nos casos em que existia dúvida relativamente ao género do candidato, foi usado o motor de busca Google para obter uma identificação de género através da pesquisa de fotografia do candidato e reconhecimento de traços fenotípicos caraterísticos de género. Com base na identificação de género, procedeu-se ao cálculo da percentagem de mulheres candidatas a nível nacional, por secção, por lista, e por cargo a que os indivíduos se candidatavam. Visto que os cargos apresentavam diferenças entre as várias listas, para a análise por cargo apenas se analisaram os cargos comuns a todas as listas.

De forma a estimar o que seria esperado de acordo com o número de médicas, foram calculadas razões observado-versus-esperado (O/E) por secção regional e intervalos de confiança a 95\% assumindo uma distribuição de Poisson. Os valores observados foram os utilizados para calcular a proporção de candidatas. Os valores esperados foram obtidos aplicando a proporção de médicos do sexo feminino ${ }^{8}$ ao número total de candidatos em cada secção regional. Dado que a proporção de médicos do sexo feminino tem vindo a aumentar, e é expectável que os candidatos tenham já alguma experiência, foram utilizadas duas proporções - a do ano de 2009 e de 2016 . O número esperado com base de 2009 dá-nos o número de médicos do sexo feminino caso o conjunto de candidatos seguisse a distribuição de género existente em 2009. De igual modo, o número esperado com base em 2016 dá-nos o que seria expectável caso o conjunto de candidatos seguisse a distribuição de género existente em 2016. Uma razão inferior a 1 significa que existem menos candidatos do sexo feminino do que seria esperado de acordo com a distribuição de médicos por género, um valor de 1 corresponde ao valor esperado, e acima de 1 superior ao valor esperado.

Dado que os candidatos a suplentes têm uma menor possibilidade de ter desempenho efetivo do cargo, foram realizadas análises de sensibilidade excluindo os candidatos a suplentes. As análises foram conduzidas com recurso ao Microsoft Excel (versão 2016, Microsoft, Redmond, Washington, EUA) e R. ${ }^{14}$

Dado que foram utilizados apenas dados existentes no domínio público o trabalho não foi submetido para aprovação de Comissão de Ética.

\section{RESULTADOS}

Foi extraída informação relativa a 214 candidatos de todas as listas das três secções regionais. Destes, 78 eram 
do sexo feminino (aproximadamente 37\%). Relativamente às secções Norte, Centro e Sul, as percentagens de candidatos do sexo feminino foram $51 \%, 46 \%$ e $29 \%$, respetivamente. A distribuição por listas, divididas por secção regional, encontra-se apresentada na Tabela 1, evidenciando que das seis listas apresentadas apenas uma tinha metade ou mais de mulheres.

Os resultados da análise das razões observados-versus-esperados são apresentados na Tabela 2. Verifica-se que o número de candidatas a nível nacional é inferior ao esperado, tendo em consideração a distribuição de médicas. No Norte, a distribuição é concordante com o esperado enquanto que em Lisboa o número de candidatas é quase metade do esperado. Os resultados são semelhantes considerando a distribuição de médicas em 2009 e 2016. Quando são retirados os candidatos a suplentes, as razões diminuem em todos os grupos.

$\mathrm{Na}$ Tabela 3 é apresentada a percentagem de candidatas por posição. Nos cargos identificados como presidente (Mesa da Assembleia Regional, Conselho Regional e Conselho Fiscal Regional) existia um candidato do sexo feminino, num total de 18 candidatos. De forma semelhante, para a posição de vice-presidente de lista, 33\% dos candidatos eram mulheres. No que diz respeito ao cargo de tesoureiro, apenas uma candidatura (17\%) apresentava mulheres. Num total de seis mandatários e seis delegados de lista, apenas existiu um nome de mulher em cada uma destas funções. No caso da Secção Regional Sul, nenhuma mulher surge como mandatária, delegada ou candidata a presidente de órgão. Por outro lado, no que diz respeito às posições de secretário e de suplente para os diferentes órgãos, $56 \%$ e $54 \%$ dos candidatos eram mulheres.

\section{DISCUSSÃO}

Este estudo constitui a primeira caracterização conhecida da desigualdade de género em situação de candidaturas a cargos de liderança na área médica em Portugal. Os resultados apresentados demonstram uma menor participação de médicas no processo eleitoral do que o esperado, considerando o número de médicas. Esta diferença torna-se ainda mais relevante quando retiramos candidaturas a cargos suplentes.

A diferença entre géneros é particularmente acentuada na região Sul, sendo o número de candidatas cerca de metade do número esperado. Contudo, é de realçar que na Secção Regional Norte, o aparente melhor resultado se deve a uma lista ter uma elevada percentagem de candidatas. Esta lista incluía o aumento do número de mulheres em posições efetivas na sua missão de campanha eleitoral. ${ }^{13} \mathrm{~A}$ aparente diferença geográfica deve também ser interpretada com precaução, dado os números analisados serem pequenos e por isso terem grande variabilidade. Estudos futuros poderão analisar outros exemplos de cargos de liderança e aprofundar a análise em termos de distribuição geográfica.

Em relação às diferenças de percentagens de mulheres candidatas por cargos a que se candidatam, verificou-se uma predominância de candidatos homens a assumir posi-

Tabela 1 - Candidatos do género feminino envolvidas no processo eleitoral por secção regional e lista (total e excluindo as candidaturas a cargos suplentes)

\begin{tabular}{lccc}
\hline $\begin{array}{l}\text { Secção } \\
\text { regional }\end{array}$ & Lista & $\begin{array}{c}\text { Candidatos do } \\
\text { género feminino } \\
\%(\mathrm{n}, \mathrm{T})\end{array}$ & $\begin{array}{c}\text { Candidatos do género feminino, } \\
\text { excluindo candidaturas a suplentes } \\
\%(\mathrm{n}, \mathrm{T})\end{array}$ \\
\hline \multirow{2}{*}{ Norte } & 1 & $29(7 / 24)$ & $25(5 / 20)$ \\
& 2 & $76(16 / 21)$ & $71(12 / 17)$ \\
Centro & Total & $51(23 / 45)$ & $46(17 / 37)$ \\
& 3 & $46(16 / 35)$ & $41(11 / 27)$ \\
Sul & 4 & $33(15 / 45)$ & $30(11 / 37)$ \\
& 5 & $36(16 / 45)$ & $37(14 / 38)$ \\
\hdashline$-\cdots$ & $18(8 / 44)$ & $11(4 / 36)$ \\
\hline
\end{tabular}

T: total de candidatos para cada grupo

Tabela 2 - Razões observado-versus-esperado com e sem suplentes, nos anos de 2009 e 2016 a nível nacional e pelas três secções regionais (Norte, Centro e Sul)

\begin{tabular}{lllll}
\hline & \multicolumn{2}{c}{ O/E 2009 } & \multicolumn{2}{c}{ O/E 2016 } \\
\hline Região & Com suplentes & Sem suplentes & Com suplentes & Sem suplentes \\
Nacional & $0,74(0,58 ; 0,92)$ & $0,66(0,50 ; 0,85)$ & $0,67(0,53 ; 0,84)$ & $0,60(0,45 ; 0,78)$ \\
Norte & $1,05(0,66 ; 1,57)$ & $0,94(0,55 ; 1,51)$ & $0,92(0,58 ; 1,38)$ & $0,85(0,50 ; 1,36)$ \\
Centro & $0,94(0,54 ; 1,53)$ & $0,85(0,42 ; 1,51)$ & $0,89(0,51 ; 1,44)$ & $0,79(0,39 ; 1,41)$ \\
Sul & $0,58(0,41 ; 0,80)$ & $0,52(0,35 ; 0,74)$ & $0,54(0,39 ; 0,74)$ & $0,48(0,32 ; 0,69)$ \\
\hline
\end{tabular}


Tabela 3 - Candidatos do género feminino envolvidos no processo eleitoral por posição e por região

\begin{tabular}{|c|c|c|}
\hline Posição* & $\begin{array}{l}\text { Secção } \\
\text { Regional }\end{array}$ & $\begin{array}{c}\text { Candidatos do } \\
\text { género feminino } \\
\%(n, T)\end{array}$ \\
\hline \multirow{4}{*}{ Mandatário } & Norte & $0(0 / 2)$ \\
\hline & Centro & $100(1 / 1)$ \\
\hline & Sul & $0(0 / 3)$ \\
\hline & Total & $17(1 / 6)$ \\
\hline \multirow{4}{*}{ Delegado } & Norte & $50(1 / 2)$ \\
\hline & Centro & $0(0 / 1)$ \\
\hline & Sul & $0(0 / 3)$ \\
\hline & Total & $17(1 / 6)$ \\
\hline \multirow{4}{*}{ Presidente } & Norte & $20(1 / 5)$ \\
\hline & Centro & $0(0 / 3)$ \\
\hline & Sul & $0(0 / 7)$ \\
\hline & Total & $7(1 / 15)$ \\
\hline \multirow{4}{*}{ Vice-presidente } & Norte & $50(2 / 4)$ \\
\hline & Centro & $50(1 / 2)$ \\
\hline & Sul & $17(1 / 6)$ \\
\hline & Total & $33(4 / 12)$ \\
\hline \multirow{4}{*}{ Tesoureiro } & Norte & $0(0 / 2)$ \\
\hline & Centro & $0(0 / 1)$ \\
\hline & Sul & $33(1 / 3)$ \\
\hline & Total & $17(1 / 6)$ \\
\hline \multirow{4}{*}{ Secretário } & Norte & $83(5 / 6)$ \\
\hline & Centro & $33(1 / 3)$ \\
\hline & Sul & $44(4 / 9)$ \\
\hline & Total & $56(10 / 18)$ \\
\hline \multirow{4}{*}{ Vogais } & Norte & $50(8 / 16)$ \\
\hline & Centro & $71(5 / 7)$ \\
\hline & Sul & $30(7 / 23)$ \\
\hline & Total & $42(20 / 46)$ \\
\hline \multirow{4}{*}{ Suplentes } & Norte & $75(6 / 8)$ \\
\hline & Centro & $63(5 / 8)$ \\
\hline & Sul & $44(10 / 23)$ \\
\hline & Total & $54(21 / 39)$ \\
\hline
\end{tabular}

*: Apenas se apresentam as posições com candidatos em todas as listas pelo que o somatório difere do total de candidatos.

$\mathrm{T}$ : total de candidatos para cada grupo

ções de liderança, enquanto existe um maior predomínio de mulheres a concorrer a cargos de menor exposição (como secretário) ou menor possibilidade de desempenho efetivo (suplente da lista). Existe evidência de maior predisposição para criticar detentores de cargos em posições a que o seu género não é tipicamente associado. ${ }^{15}$ Se considerarmos que cargos de liderança não estão tradicionalmente associados ao género feminino, tal pode indicar que, para além de maiores dificuldades em atingir este tipo de posições, as mulheres poderão sentir-se como foco de crítica, optando por escolher posições de menor exposição ou envolvimento menos ativo.
Para além do acima referido, entre as razões apontadas na literatura como possíveis explicações para uma menor participação das mulheres em posições de liderança encontram-se aspetos relacionados com o papel social da mulher: (i) a maternidade, sobretudo nos seus anos iniciais; (ii) a imagem social de priorização da família para as mulheres $^{10,16,17}$; (iii) as mulheres continuarem a dedicar mais tempo às tarefas domésticas do que os seus companheiros. ${ }^{7}$ Para além do papel na maternidade e nas tarefas domésticas, está descrito que as mulheres poderão ter um menor interesse em assumir posições de liderança por perceções associadas a estes cargos (e.g. possível afastamento da medicina ${ }^{9}$ e sobrevalorização de desafios associados a estes). ${ }^{18}$ Para atingir maior igualdade de género em posições de liderança, algumas organizações médicas estão a divulgar exemplos de liderança por mulheres e a alterar políticas institucionais relativas à maternidade, para facilitar a conciliação da mesma com o exercício profissional.9,10 Outra possível intervenção será a implementação de esquemas de mentoria. ${ }^{10,16}$ Embora estes aspetos requeiram medidas concertadas a vários níveis de atuação e fora do âmbito do presente trabalho, consideramos que os resultados agora apresentados contribuem para a discussão do papel das mulheres na liderança na área médica e das medidas a implementar para dar resposta à desigualdade existente.

Este estudo apresenta várias limitações que devem ser consideradas. Verifica-se a não completa sobreposição das secções da OM com as regiões obtidas a nível de NUTS II. Contudo, dada a extensão limitada da zona de não-sobreposição e a magnitude dos resultados encontrados é pouco expectável que esta limitação tenha enviesado os resultados de forma relevante. Por outro lado, este estudo apenas considerou uma eleição para alguns dos corpos da OM, reflectindo por isso uma imagem limitada da situação a nível nacional, num período eleitoral específico. Este exemplo foi selecionado devido à disponibilidade pública de dados para o realizar e constitui, graças à inexistência de estudos prévios, um primeiro contributo na caracterização da situação de desigualdade de género nos cargos de liderança na área médica, a nível nacional. Uma caracterização mais detalhada da situação e a compreensão dos motivos que a causam serão aspetos a considerar em estudos futuros. Para além disso, dada a recente obrigatoriedade de apresentação de listas candidatas mais paritárias, ${ }^{19}$ será também relevante observar de que forma esta condicionante se reflete na constituição das listas a ser apresentadas.

\section{CONCLUSÃO}

Há uma menor participação do que o que seria expectável por parte das médicas nos processos eleitorais da OM, com significância estatística a nível nacional e na Secção Regional do Sul. Quando participam, as mulheres tendem a estar mais presentes em cargos de menor relevância, ou com menor potencial para eleição (e.g. secretário ou suplente). Estes resultados indicam a necessidade de uma maior compreensão desta problemática e medidas para o combate à desigualdade de género em cargos de liderança. 


\section{PROTECÇÃO DE PESSOAS E ANIMAIS}

Os autores declaram que os procedimentos seguidos estavam de acordo com os regulamentos estabelecidos pelos responsáveis da Comissão de Investigação Clínica e Ética e de acordo com a Declaração de Helsínquia da Associação Médica Mundial revista em 2013.

\section{CONFIDENCIALIDADE DOS DADOS}

Os autores declaram ter seguido os protocolos do seu

\section{REFERÊNCIAS}

1. Equality European Institute for Gender. Gender Equality in Power and Decision-Making [e-report]. 2015. [consultado 2019 out 14]. Disponível em: http://eige.europa.eu/sites/default/files/documents/mh0215090enn. pdf\#page=1\&zoom=auto,-22,842.

2. United Nations. Gender equality. [consultado 2019 out 14]. Disponível em: https://www.un.org/en/sections/issues-depth/gender-equality/.

3. United Nations. Sustainable Development Goals - Goal 5: Achieve gender equality and empower all women and girls. [consultado 2019 ut 14]. Disponível em: https://www.un.org/sustainabledevelopment/ gender-equality/.

4. Diário de Notícias. Portugal na cauda da Europa em equilíbrio de género nas empresas. 2019. [consultado 2019 out 14]. Disponível em: https://www.dn.pt/pais/interior/portugal-a-meio-do-pelotao-a-nivel-doequilibrio-de-genero-nas-empresas---estudo-10597686.html.

5. Rodrigues MS. A representatividade das mulheres na liderança de topo: análise das atuais empresas do PSI-20 (2005 a 2016). 2017. [consultado 2019 out 14]. Disponível em: https://repositorio-aberto. up.pt/bitstream/10216/108193/2/224382.pdf.

6. Alves H. Gender equity in the medical profession as a democratic culture: the Portuguese experience. In: Bellini MI, Papalois V, editors. Gender equity in the medical profession. Hershey: Medical Information Science Reference; 2020. p. 199-2013.

7. Hitti E, Faaem M, Moreno-Walton L, Faaem M. The gender gap in medical leadership: glass ceiling, domestic tethers, or both? American Academy Emergency Medicine News. 2017. [consultado 2019 out 14]. Disponível em: https://www.aaem.org/UserFiles/file/MayJune17_WiEM. pdf.

8. PORDATA. Médicos: total e por sexo. 2019 [consultado 2019 centro de trabalho acerca da publicação de dados.

\section{CONFLITOS DE INTERESSE}

Os autores declaram não ter conflitos de interesse relacionados com o presente trabalho.

\section{FONTES DE FINANCIAMENTO}

Este projeto não recebeu qualquer apoio financeiro.

\section{out 14]. Disponível em: https://www.pordata.pt/Portugal/} Médicos+total+e+por+sexo-1966.

9. Glauser W. Rise of women in medicine not matched by leadership roles. CMAJ. 2018;190:E479-80.

10. Boylan J, Dacre J, Gordon H. Addressing women's under-representation in medical leadership. Lancet. 2019;393:e14.

11. Machado MC. A feminização da medicina. Análise Social. 2003;38:12737.

12. Ordem dos Médicos. História da Ordem. [consultado 2019 out 14]. Disponível em: https://ordemdosmedicos.pt/historia-da-ordem/.

13. Ordem dos Médicos. ROM. 2016;32:81-93.

14. RStudio Team. RStudio: Integrated Development for R. 2016. [consultado 2019 out 14]. Disponível em: http://www.rstudio.com/.

15. Brescoll VL, Dawson E, Uhlmann EL. Hard won and easily lost. Psychol Sci. 2010;21:1640-2.

16. Mangurian C, Linos E, Sarkar U, Rodriguez C, Jagsi R. What's holding women in medicine back from leadership. Harv Bus Rev. 2018. [consultado 2019 out 14]. Disponível em: https://hbr.org/2018/06/whatsholding-women-in-medicine-back-from-leadership.

17. Jolly S, Griffith KA, DeCastro R, Stewart A, Ubel P, Jagsi R. Gender differences in time spent on parenting and domestic responsibilities by high-achieving young physician-researchers. Ann Intern Med. 2014;160:344-53

18. Cajigal S, Weiss G, Silva N. Women as physician leaders. Medscape. 2015. [consultado 2019 out 14]. Disponível em: https://www.medscape. com/features/slideshow/public/femaleleadershipreport2015.

19. Lei n. ${ }^{\circ}$ 26/2019. Diário da República, I Série, n. ${ }^{\circ} 62$ (2019/03/28) p.1751-2. 